\title{
Calculation on the Ion Flow Field under HVDC Transmission Lines Considering Wind Effects
}

\begin{abstract}
Jing Wu ${ }^{\dagger}$, Sheng Ga** and Yuxiao Liu*
Abstract - Based on Deutsch assumption, a calculation method on the electric field over the ground surface under HVDC transmission lines in the wind is proposed. Analyzing the wind effects on the electric field and the space charge density the existing method based on Deutsch assumption is improved through adding the wind speed to the ion flow field equations. The programming details are illustrated. The calculation results at zero wind speed are compared with available data to validate the code program. Then the ionized fields which resulted from corona of $\pm 800 \mathrm{kV}$ HVDC lines are analyzed. Both the electric field and the current density on the ground level are computed under different wind direction and speed. The computation results are in good agreement with measurements. The presented method and code program can be used to rapidly predict and evaluate the wind effects in HVDC transmission engineering.
\end{abstract}

Keywords: Corona, Deutsch assumption, HVDC transmission line, Ion flow field, Wind effects

\section{Introduction}

Corona discharges usually occur around the high voltage overhead transmission lines. The space charges created by AC corona are constrained to the vicinity of conductors because of the periodical reversal of electric field. However, the charges created by DC corona fill in the entire space and drift toward either the conductor of opposite polarity or the ground plane. If the space charges move to the ground plane, the ground-level electric field will be enhanced and can be several times larger than the space-charge-free electric field. Such fields are called ion flow fields.

In the design and construction of high voltage direct current (HVDC) transmission lines, it is necessary to control the electric field over the ground surface and make it satisfy the environment protection requirements [1]. The calculation on the electric field has received wide attentions [2-15] involving the methods based on Deutsch assumption [2-7] and the mesh-based methods [8-14]. Deutsch assumption supposes that space charges affect only the magnitude of the electric field without changing its direction. In the calculation, the Deutsch assumption based methods avoid mesh division and complex computations and they have fast calculation speed. They have been applied to double-circuit HVDC transmission lines on the same tower in china [4] and achieved engineering benefits. Because the geographical and climatic conditions of the regions covered by the overhead transmission lines are various, they should not be

$\dagger$ Corresponding Author: School of Automation Science and Electrical Engineering, Beihang University, Beijing, China.

(wujing06@buaa.edu.cn)

* School of Automation Science and Electrical Engineering, Beihang University, Beijing, China. (\{gaoshenghsg, txbj2011\}@163.com)

Received: November 13, 2014; Accepted: March 31, 2015 neglected in analyzing accurately the electric fields around the lines. As for the existing mesh-based methods, the air factors such as the wind [10, 11], the rainfall [12] and the fog [13] have been taken into account. However, they were ignored in the Deutsch assumption based methods.

In this paper, we propose a Deutsch assumption based method to solve the bipolar DC ion flow field within the wind. The equations and boundary conditions describing the ion flow field are presented. A program is coded to solve the boundary value problem. The effectiveness of the program is validated by some measurement results. Finally some characteristics of ion flow field considering the wind speed and direction are given.

\section{Equations Considering Wind Effects}

The ion flow field of HVDC transmission lines is assumed as follows [2-7]

(1) The field is two-dimensional.

(2) Drifting particles are positive around the positive conductor and negative around the negative one. The particles over the ground surface are either positive or negative and the ion flow field is unipolar.

(3) The thickness of the ion layer is negligible, and the electric field at the conductor surface remains constant which is the corona onset value.

(4) The diffusion of the ions is neglected, and the ion mobility keeps constant.

The equations on the electric field $\boldsymbol{E}_{s}$ and the ion current density $\boldsymbol{J}$ when the corona appears are described as

$$
\nabla \cdot \mathbf{E}_{s}=\rho / \varepsilon_{0}
$$




$$
\begin{gathered}
\mathbf{J}=\rho\left(K \mathbf{E}_{s}+\mathbf{w}\right) \\
\nabla \cdot \mathbf{J}=0
\end{gathered}
$$

where $\rho$ is the space charge density, $w$ is the wind speed, $\varepsilon_{0}$ is the permittivity of air, and $K$ is the ion mobility.

The above equations describe a positive ionized field. In the region filled with negative charges, Eq. (2) can be rewritten as $\mathbf{J}=\rho\left(-K \mathbf{E}_{s}+\mathbf{w}\right)$.

The space charges in the ion flow field move along the electric flux lines. The moving speed is $\mathbf{v}=K \mathbf{E}_{s}$. Generally, $K$ is in the range of $(1.2 \sim 2.0) \times 10^{-4} \mathrm{~m}^{2} /(\mathrm{V} \cdot \mathrm{s})$, and $\boldsymbol{E}_{s}$ is in $(0 \sim 50) \mathrm{kV} / \mathrm{m}$, so $\boldsymbol{v}$ is in $(0 \sim 10) \mathrm{m} / \mathrm{s}$.

Moreover, according to (2) the wind may well change the ion current density through changing the moving speed of space charges as similar as $\boldsymbol{E}_{s}$ does. Historically, Beaufort wind force scale provides an empirical description of wind speed based on observed sea conditions. Table 1 illustrates the wind speed and the ground state from level 0 to 7. Apparently, the wind speed is comparable with the charge moving speed in the ion flow field. Thus its effects should not be ignored.

Table 1. Wind classification

\begin{tabular}{c|c|c|l}
\hline Level & Description & Speed/(m/s) & \multicolumn{1}{|c}{ Ground states } \\
\hline 0 & Calm & $0.0-0.2$ & Smoke rising vertically. \\
\hline 1 & Light air & $0.3-1.5$ & $\begin{array}{l}\text { Smoke drift indicating wind } \\
\text { direction. }\end{array}$ \\
\hline 2 & Light breeze & $1.6-3.3$ & Wind felt on exposed skin. \\
\hline 3 & Gentle breeze & $3.4-5.4$ & $\begin{array}{l}\text { Leaves and small twigs constantly } \\
\text { move, and light flags are extended. }\end{array}$ \\
\hline 4 & $\begin{array}{c}\text { Moderate } \\
\text { breeze }\end{array}$ & $5.5-7.9$ & $\begin{array}{l}\text { Dust and loose paper are raised, and } \\
\text { small branches begin to move. }\end{array}$ \\
\hline 5 & Fresh breeze & $8.0-10.7$ & Small trees in leaf begin to sway. \\
\hline 6 & Strong breeze & $10.8-13.8$ & $\begin{array}{l}\text { Whistling is heard in overhead } \\
\text { wires. }\end{array}$ \\
\hline 7 & High wind & $13.9-17.1$ & $\begin{array}{l}\text { Whole trees are in motion. Efforts } \\
\text { are needed to walk against the wind. }\end{array}$ \\
\hline
\end{tabular}

\section{Solving Method on the Field}

\subsection{Boundary value problem}

According to the Deutsch assumption, the space charges affect only the magnitude of the electric field rather than its direction. Therefore,

$$
\mathbf{E}_{s}=A \mathbf{E}
$$

where $\boldsymbol{E}$ is the space-charge-free electric field, $A$ is a scalar function depending on the distribution of space charges.

$\boldsymbol{E}$ and $\boldsymbol{E}_{s}$ can be expressed by the space-charge-free potential $\varphi$ and the potential $\phi$ in the presence of space charges

$$
\begin{gathered}
\mathbf{E}=-\nabla \varphi \\
\mathbf{E}_{s}=-\nabla \phi
\end{gathered}
$$

The field flux lines are not changed by the presence of space charges in Deutsch assumption, so

$$
\begin{aligned}
& E=-\mathrm{d} \varphi / \mathrm{d} s \\
& E_{s}=-\mathrm{d} \phi / \mathrm{d} s
\end{aligned}
$$

where $s$ is the distance along the flux line of the spacecharge-free field.

From (4), (7) and (8),

$$
\mathrm{d} \phi / \mathrm{d} \varphi=A
$$

Substitute (4) into (1), and then (noting that $\nabla \cdot \mathbf{E}=0$ )

$$
\mathbf{E} \cdot \nabla A=\rho / \varepsilon_{0}
$$

Replacing $\mathbf{E} \cdot \nabla A$ by $E \mathrm{~d} A / \mathrm{d} s=\rho / \varepsilon_{0}$ and according to the relation:

$$
\frac{\mathrm{d} A}{\mathrm{~d} s}=\frac{\mathrm{d} A}{\mathrm{~d} \varphi} \frac{\mathrm{d} \varphi}{\mathrm{d} s}=-E \frac{\mathrm{d} A}{\mathrm{~d} \varphi}
$$

Eq. (10) is transformed into

$$
\frac{\mathrm{d} A}{\mathrm{~d} \varphi}=-\frac{\rho}{\varepsilon_{0} E^{2}}
$$

The space charge density along the flux line may also be derived in a similar way. From (2) and (3),

$$
\nabla \cdot\left(\rho K \mathbf{E}_{s}+\mathbf{w}\right)=\nabla \cdot\left(\rho K \mathbf{E}_{s}\right)+\nabla \cdot(\rho \mathbf{w})=0
$$

where

$$
\nabla \cdot\left(\rho K \mathbf{E}_{s}\right)=K\left(\rho^{2} / \varepsilon_{0}-A E^{2} \mathrm{~d} \rho / \mathrm{d} \varphi\right)
$$

and

$$
\nabla \cdot(\rho \mathbf{w})=\mathbf{w} \cdot \nabla \rho+\rho \nabla \cdot \mathbf{w}=-\frac{\mathrm{d} \rho}{\mathrm{d} \varphi} \mathbf{w} \cdot \mathbf{E}+\rho \nabla \cdot \mathbf{w}
$$

Therefore,

$$
\frac{\mathrm{d} \rho}{\mathrm{d} \varphi}=\frac{K \rho^{2} / \varepsilon_{0}+\rho \nabla \cdot \mathbf{w}}{K A E^{2}+\mathbf{w} \cdot \mathbf{E}}
$$

Eqs. (9), (11) and (12) describe the distribution of the electric field and the charges within the wind. When the wind speed $\boldsymbol{w}$ is set to 0 , these equations are in agreement with those without the wind [14]. The boundary conditions required to solve these equations are given as follows

$$
\left\{\begin{array}{c}
\varphi=0, \phi=0 \\
\varphi=V, \phi=V \quad \text { on the ground level } \\
A=A_{e}=V_{0} / V \quad \text { at the conductor surface }
\end{array}\right.
$$


where $V_{0}$ is the onset voltage of corona, $A_{e}$ is the value of the scalar function $A$ at the conductor surface.

The boundary value problem defined by (9), (11), (12) and (13) has no analytical solutions and it should be solved numerically.

\subsection{Programming method}

Referring to the program described in [4] which has been validated and now widely used in electric power engineering survey and design in China, a program solving the boundary value problem considering wind effects is coded to calculate the electric field over the ground surface under HVDC transmission lines shown in Fig. 1. $U$ is the voltage applied to the conductors, $N$ is the number of subconductors of each bundle conductor, $R$ is the radius of each subconductor, $D$ is the bundle span, $L$ is the polar span and $H$ is the height of the bundle conductor axial. The programming details are described as follows.

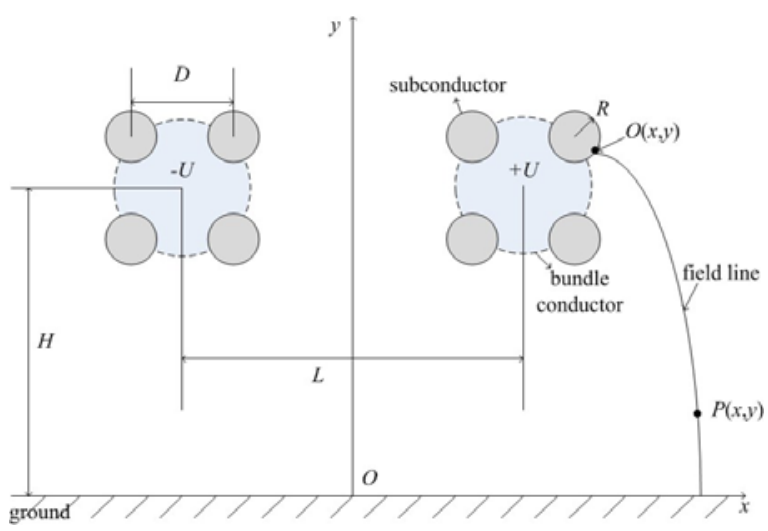

Fig. 1. Configuration of the transmission lines

1) Calculation on the Space-Charge-Free Electric Field: The bundled conductors are usually used in HVDC transmission lines. We use the successive image method to calculate their space-charge-free electric fields as in [16].

2) Calculation on the Electric Field Lines: In the Deutsch assumption based methods, the calculation on the electric field strength and the charge density is along the electric field lines [2, 3]. The electric field at any point outside the pole wires satisfies

$$
\mathrm{d} x / E_{x}=\mathrm{d} y / E_{y}
$$

where $E_{x}$ and $E_{y}$ are the field strengths in x- and ydirection.

Suppose a point $P(x, y)$ is on the electric field line and its close point is $P^{\prime}\left(x^{\prime}, y^{\prime}\right)$. Taking the length $\Delta l=\sqrt{(\Delta x)^{2}+(\Delta y)^{2}}$ along the field line as a step the coordinates of $P^{\prime}$ are expressed as

$$
\left\{\begin{array}{l}
x^{\prime}=x+\lambda \Delta l \frac{E_{x}}{\sqrt{E_{x}^{2}+E_{y}^{2}}} \\
y^{\prime}=y+\lambda \Delta l \frac{E_{y}}{\sqrt{E_{x}^{2}+E_{y}^{2}}}
\end{array}\right.
$$

where $\lambda=1$ when $P^{\prime}$ is along the field direction and $\lambda=-1$ when it is along the opposite field direction.

3) Calculation on the Corona Onset Voltage: The corona onset field strength $E_{\text {on }}$ of each subconductor in the transmission line is calculated by the formula in [17]

$$
E_{\text {on }}=30 m\left(1+\frac{0.301}{\sqrt{R}}\right)
$$

where $E_{\text {on }}$ is measured by $\mathrm{kV} / \mathrm{cm}, m(0<m \leq 1)$ is the roughness coefficient of the conductor surface which reflects the environment influence on corona discharge, and $R(\mathrm{~cm})$ is the subconductor radius. Eq. (16) is obtained by modifying the empirical formula proposed in [18] according to the practical transmission lines in China and $m$ is taken as 0.5 by many tests.

When the maximal field strength $E_{\max }$ of each subconductor surface reaches to $E_{\text {on }}$, the corresponding voltage is the corona onset voltage. From (4), the scalar function at each pole surface is

$$
A_{\mathrm{e}}=\left|\frac{E_{\mathrm{on}}}{\bar{E}_{\mathrm{max}}}\right|
$$

where $\bar{E}_{\max }$ is the average value of $E_{\max }$. If the transmission line uses two bundle conductors, and the maximal field strengths on their surfaces are $E_{\max _{1}}$ and $E_{\max _{2}}$, then $\bar{E}_{\max }=\left(E_{\max _{1}}+E_{\max _{2}}\right) / 2$. The corona onset voltage is described as $V_{0}=A_{e} V$, where $V$ is the voltage applied to the transmission line.

4) Calculation on the Electric Field over the Ground Surface: To obtain the electric field at $P(x, y)$ over the ground surface, (9), (11) and (12) are solved by the secant method.

a) Calculate the potential at $P(x, y)$ and determine the electric field line through this point and the intersection point $O(x, y)$ of this line and the pole conductor surface. $A_{e}$ at $O(x, y)$ is calculated by (17).

b) Two initial values of the charge density at $O(x, y)$ are chosen as $\rho_{e 1}$ and $\rho_{e 2}$

$$
\left\{\begin{array}{l}
\rho_{e 1}=f_{1} \rho_{m} \\
\rho_{e 2}=f_{2} \rho_{m}
\end{array}\right.
$$

where $f_{1}$ and $f_{2}$ are positive constants and their 
values can be chosen freely. Here we adopt $f_{1}=1.5$ and $f_{2}=3$ which can make the computation have a faster convergence. $\rho_{m}$ is the average charge density [2].

$$
\rho_{m}=\frac{\int_{0}^{V} \int_{\varphi}^{V} E^{-2} \rho \mathrm{d} \eta \mathrm{d} \varphi}{\int_{0}^{V} \int_{\varphi}^{V} E^{-2} \mathrm{~d} \eta \mathrm{d} \varphi}=\frac{\varepsilon_{0}\left(V-V_{o}\right)}{\int_{0}^{V} \int_{\varphi}^{V} E^{-2} \mathrm{~d} \eta \mathrm{d} \varphi}
$$

c) For the initial value $\rho_{e 1}$, taking $O(x, y)$ as a start point and considering the boundary condition (13), the two order of Runge-Kutta method is used to solve (9), (11) and (12).

Finally, the corresponding space-charge-free potential $\varphi_{p_{1}}^{\prime}$ at $P(x, y)$ is obtained. Similarly, $\varphi_{p_{2}}^{\prime}$ corresponding to $\rho_{e 2}$ is also obtained.

d) The new estimated value $\rho_{e 3}$ is calculated by the secant iteration method and its general iteration form is

$$
t_{k+1}=t_{k}-\frac{g\left(t_{k}\right)}{g\left(t_{k}\right)-g\left(t_{k-1}\right)}\left(t_{k}-t_{k-1}\right)
$$

where let $g\left(\rho_{e k}\right)=\varphi_{p k}^{\prime}-\varphi_{p} \quad, \quad t_{k}=\rho_{e k}$ $(k=1,2,3 \cdots)$, thus

$$
\rho_{e 3}=\rho_{e 2}+\frac{\varphi_{p}-\varphi_{p_{2}}^{\prime}}{\varphi_{p_{2}}^{\prime}-\varphi_{p_{1}}^{\prime}}\left(\rho_{e 2}-\rho_{e 1}\right)
$$

e) According to c), $\varphi_{p_{3}}^{\prime}$ and $A_{p_{3}}$ corresponding to $\rho_{e 3}$ are obtained. If the following equations are satisfied, then $\rho_{e 3}$ is the estimated true value of charge density

$$
\begin{gathered}
\left|\frac{\varphi_{p}-\varphi_{p_{3}}^{\prime}}{\varphi_{p}}\right|<10^{-6} \\
\left|A_{p_{3}} \frac{\varphi_{p_{3}}^{\prime}-0}{\phi_{p_{3}}-0}-1\right|<10^{-6}
\end{gathered}
$$

Otherwise, let $\rho_{e 1}=\rho_{e 2}$ and $\rho_{e 2}=\rho_{e 3}$ and repeat 3) 5) until both (22) and (23) are satisfied at the same time.

f) Using the true value of the charge density, the electric field and the ion flow density at $P(x, y)$ are calculated out by (1) (3).

\section{Results and Discussion}

Using the above programming method, a program has

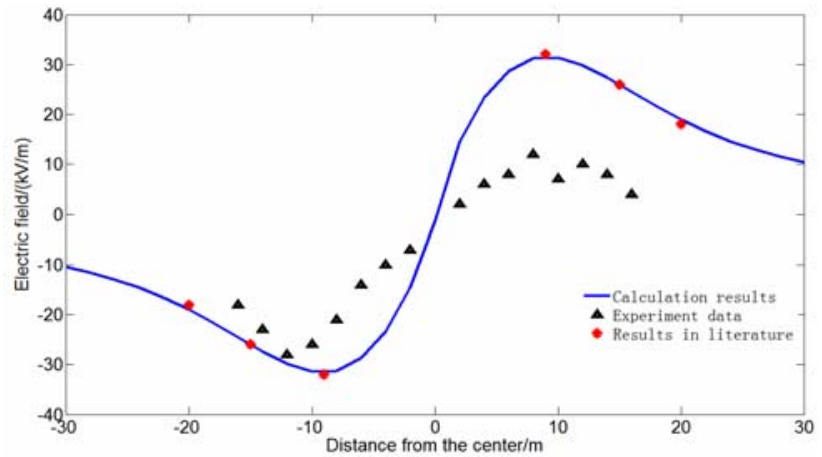

Fig. 2. Comparison on the calculation results and experimental data without wind

been coded by MATLAB. By setting $\mathbf{w}=\mathbf{0}$, the program is used to calculate the model in Fig. 1 where $U=500 \mathrm{kV}$, $R=2.37 \mathrm{~cm}, N=4, D=45 \mathrm{~cm}, L=14 \mathrm{~m}$ and $H=12.7 \mathrm{~m}$. The calculation results on the electric field over the ground surface are shown in Fig. 2, which are in agreement with the results in literature [14], but are a bit different from experimental data. This is mainly attributed to neglecting the different corona characteristics of positive and negative conductors. In addition, the accuracy of experimental data is limited by the test equipment and the environment conditions.

Inclusive of the wind, the calculation results are compared with the experimental data of $\pm 800 \mathrm{kV} \mathrm{HVDC}$ transmission lines at the National Engineering Laboratory for Ultra High Voltage Technology (Kunming) in China Southern Power Grid. $R=1.68 \mathrm{~cm}, N=6, D=45 \mathrm{~cm}, L=22 \mathrm{~m}$ and $H=18 \mathrm{~m}$. Fig. 3 and 4 show the calculation results on the electric field and the ion current density on the ground level.

In the experiments of measuring the fields, the wind speed and direction were not very stable. The wind speed was kept in the range of $1-3 \mathrm{~m} / \mathrm{s}$. The wind remained nearly the same direction blowing from the negative pole to the positive one in two hours. The measured values shown in Fig. 3 at the same point varied randomly.

From Fig. 3, when the wind direction changes, the electric field at a distance of more than 20 meters from the center varies greatly. When the wind blows from '-' pole to ' + ' pole, $\mathbf{w}$ is assumed to be positive, and we find that the absolute values of the calculated fields under ' + ' and

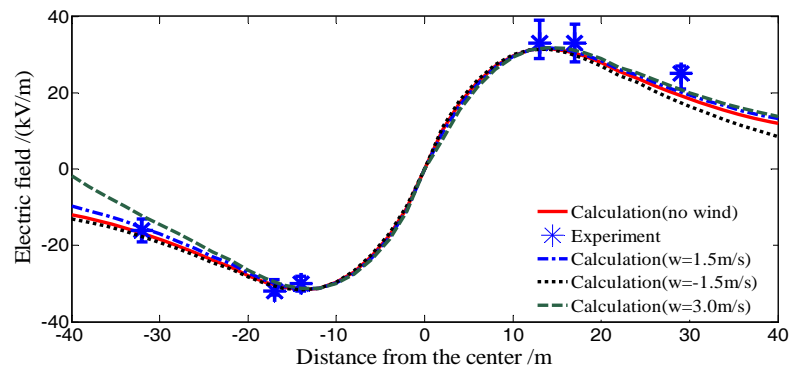

Fig. 3. Calculation results and experimental data within wind 


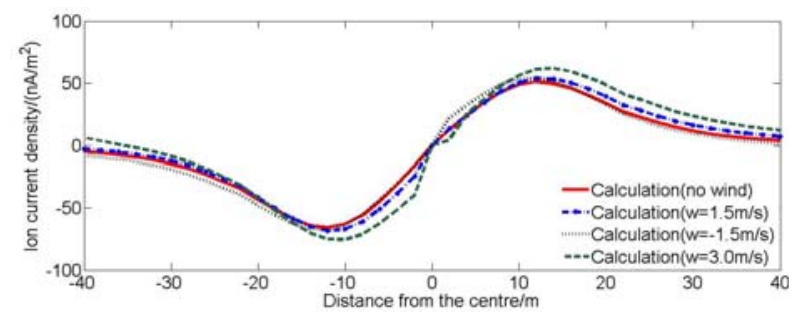

Fig. 4. Calculation results on the ion current density

'-' poles have different variation tendency. They become larger under '+' pole and smaller under '-' pole with the increase of the wind speed. When the wind direction reverses, the variation tendency is opposite to the situations above.

From Fig. 4, the variation of ion current density is similar to the electric field. However, when the value of wind speed changes, the maximum value of ion current density varies more significantly, which goes upward due to the increase of wind speed value.

\section{Conclusions}

We have presented the boundary value problems based on the Deutsch assumption and coded the calculation program on the electric field and the ion flow density caused by HVDC transmission lines in the wind. The conclusions are as follows.

(1) The wind has little effect on the electric field within a certain distance from the center of the transmission lines, while the ion current density on the ground level is easier to be affected by wind. And ion current density tends to grow with the increase of wind speed.

(2) On the lateral sides from the center, the effects of wind speed and direction on the electric field are different under positive and negative conductors. As an external force, the wind tends to move the space charges. The ion current density decreases under the negative pole and increases under the positive pole.

(3) The research work in this paper can also be expanded to evaluate other environment effects concerning dust and atmospheric pressure on HVDC transmission line by the method based on Deutsch assumption.

\section{References}

[1] Z. Y. Sun, L. S. Zeng and J. Y. Lu, “Guide for HVDC overhead transmission lines, DL/T436-2005,” Power industry standard of the People's Republic of China Tech, Nov. 1, 2005.

[2] P. S. Maruvada and W. Janischewskyj, "Analysis of corona losses on DC transmission lines: I - unipolar lines,” IEEE Trans. Power Apparatus and Systems, vol. 88, pp.718-731, 1969.

[3] P. S. Maruvada and W. Janischewskyj, “Analysis of corona losses on DC transmission lines: II - bipolar lines,” IEEE Trans. Power Apparatus and Systems, vol. 88, pp.1476-1491, 1969.

[4] Y. Yang, J. Y. Lu and Y. Z. Lei, “A calculation method for the electric field under double-circuit HVDC transmission lines," IEEE Trans. Power Delivery, vol. 23, pp.1736-1742, 2008.

[5] Y. Yang, J. Y. Lu and Y. Z. Lei, “A calculation method for the hybrid electric field under UHVAC and UHVDC transmission lines in the same corridor," IEEE Trans. Power Delivery, vol. 25, pp.1146-1153, 2010.

[6] General Electric Company, "EL-2419 HVDC transmission line research,” EPRI, 1982.

[7] T. Zhao, S. A. Sebo and D. G. Kasten, "Calculation of single phase AC and monopolar DC hybrid corona effects,” IEEE Trans. Power Delivery, vol. 11, pp. 1454-1463, 1996.

[8] W. Janischewskyj and G. Gela, "Finite Element Solution for Electric Fields of Coronating DC Transmission Lines,” IEEE Trans. Power Apparatus and Systems, vol. 98, pp. 1000-1012, 1979.

[9] T. Takuma, I. Tsutomu and K. Tadashi, "Calculation of ion flow fields of HVDC transmission lines by the finite element method,” IEEE Trans. Power Apparatus and Systems, vol. 100, pp. 4802-4810, 1981.

[10] A. Shemshadi, K. Niayesh and A. Akbari, "Influence of the airflow speed along transmission lines on the DC corona discharge loss, using finite element approach,” Physics of Plasmas, vol. 19, pp. 073506073515, 2012.

[11] H. Yin, B. Zhang, J. He and R. Zeng, “Time-domain finite volume method for ion-flow field analysis of bipolar high-voltage direct current transmission lines,” IET Generation, Transmission \& Distribution, vol. 6, pp.785-791, 2012.

[12] M. Brahami, A. Gourbi and A. Tilmatine, "Numerical analysis of the induced corona vibrations on highvoltage transmission lines affected by rainfall," IET Generation, IEEE Trans. Power Delivery, vol. 26, pp.617-624, 2011.

[13] Y. S. Zhao and W. L. Zhang, "Effects of fog on ion flow field under HVDC transmission lines," Proceedings of the CSEE, vol. 33, pp. 194-199, 2013.

[14] Y. Yang, “Analysis and calculation of the electric field under Ac and DC transmission lines in the same transmission corridor,” Beihang University, Beijing, China, 2008.

[15] P. S. Maruvada, "Electric field and ion current environment of HVDC Transmission lines: comparison of calculations and measurements," IEEE Trans. Power Delivery, vol. 27, pp. 401-410, 2012.

[16] P. S. Maruvada, "Electrostatic field of a system of 
parallel cylindrical conductors,” IEEE Trans. Power Apparatus and Systems, vol. 88, pp. 1069-1079, 1969.

[17] M. A. Salam and D. Shamloul, "Computation of ionflow fields of ac coronating wires by charge simulation techniques,” IEEE Trans. Electrical Insulation, vol.27, pp. 352-361, 1992.

[18] M. P. Sarma, "Corona performance of high-voltage transmission lines.” New York: Research Studies Press, pp. 82-83, 2000.

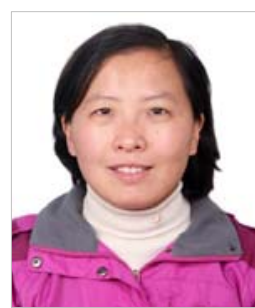

Wu Jing She received her Ph.D in Electrical Engineering from Tsinghua University, Beijing, China in 2002. She is working as Associate Professor in School of Automation Science and Electrical Engineering at Beihang University, Beijing, China. Her research interests include the electromagnetic environment of high voltage transmission engineering and the design on the electric machines and apparatus.

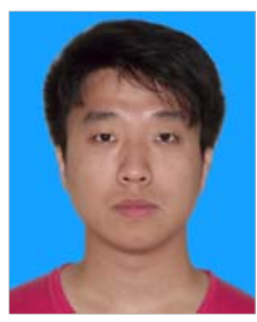

Sheng Gao He received his B. S degree in Electrical Engineering from Beihang University, Beijing, China in 2013. He is currently a Master candidate in Electrical Engineering at Beihang University, China. His research interests include high-voltage corona discharge characteristic and electric field calculation.

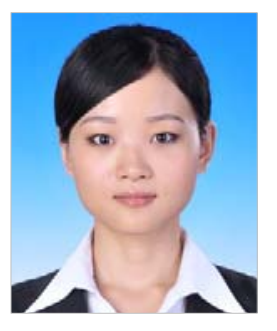

Yuxiao Liu She received her M. S. degree in Electrical Engineering from Beihang University, Beijing, China in 2014. Her research interests include high-voltage corona discharge characteristic, electric field calculation, ion mechanism in special environment, and nonlinear control. 